\title{
The Relationship between Personality and Value-Based Decision-Making
}

\author{
Fabiana Freitas Mendes \\ Faculty of Information Technology Electrical Engineering, University of Oulu \\ Oulu, Finland \\ Faculty UnB Gama, University of Brasilia \\ Gama-DF, Brazil, \\ fabiana.mendes@oulu.fi,fabianamendes@unb.br
}

\begin{abstract}
Within the context of software engineering, many decisions take place and such decisions should employ value propositions that focus on short as well as long-term goals. In 2003, Boehm coined the term Value-Based Software Engineering (VBSE), which entails the change from a value-neutral to a value-based approach. VBSE argues that decisions should be based on all key stakeholders' value propositions, and to balance both reach the short as well as longterm goal(s) the ones. This paper details a $\mathrm{PhD}$ research plan that aims to study the relationship between personality and decisionmaking within the context of VBSE. The research focuses on group decision-making, considering three aspects: the interaction among decision-makers, their perception of the decision value and their personality traits. The research methodology will be experiment which will revolve around a hypothetical software development project and some decisions that need to be made, for example, the features to be included and the priority of each one. The contribution from a theoretical perspective is to understand the relationship among three main aspects: personality traits, decision-making process, and decision value. From practitioners' perspective, the contribution is to provide help on improving software project decisionmaking.
\end{abstract}

\section{CCS CONCEPTS}

- Social and professional topics; • Professional Topics; • Management of computing and information systems; • Project and people management;

\section{KEYWORDS}

Value-Based Software Engineering, Personality, Decision-Making

ACM Reference Format:

Fabiana Freitas Mendes. 2018. The Relationship between Personality and Value-Based Decision-Making. In ICSE '18 Companion: 40th International Conference on Software Engineering , May 27-fune 3, 2018, Gothenburg, Sweden. ACM, New York, NY, USA, 2 pages. https://doi.org/10.1145/3183440. 3183444

\section{INTRODUCTION}

Software Engineering (SE) has been plagued with numerous valueneutral approaches for managing and developing software, where all requirements, defects etc., are taken as of equal importance, and where decisions are based only upon short-term goals such as cost and schedule [2]. Such "blinked-view" motivated Boehm to proposed and set an agenda for what he coined Value-Based Software Engineering (VBSE)[2]. VBSE aims to bring a balance between short- and long-terms goals where decisions are made not only by development teams, but also by other key stakeholders (for example, customers, and marketing). In such a context, the decision-making with the participation of all key stakeholders is taken as one of the main pillars [3].

One of the pillars of VBSE is the participation of all key stakeholders in all project decisions [2]. Furthermore, since decisions are made for human beings, human factors (personality for example) are important. Regarding the discussion of VBSE and decisionmaking, among the 101 papers identified in [6], only five relate to decision-making (DM), and none of them discusses the relationship between personality and DM. Additionally, considering the relationship between personality and other subjects within an SE context, among the 90 articles identified in [4], none of them focused on personality and decision-making, or personality and VBSE simultaneously.

Despite the lack of research on SE field that discusses the relationship between decision-making and personality, this relationship has been the focus of studies available outside SE domain. The literature review presented in [1] identified 29 publications that discuss the influence of personality on top management strategical decisionmaking. The authors classified the results considering a widely-used personality trait framework: Five-Factor Model. Considering this model, most of the articles concentrated on emotional stability factor (41\%), followed by extroversion and conscientiousness (30\% each one), openness to experience (15\%) and agreeableness (11\%). Note that the sum of the percentages is more than $100 \%$ because one paper can discuss more than one personality factor simultaneously.

This $\mathrm{PhD}$ research aims to address such gap by investigating the relationship between personality and group decision-making on VBSE context. To achieve such goal, there are three specific goals: -SG1: Investigate the relationship between personality and decisionmaking process.

- SG2: Investigate the relationship between decision-making process and decision value.

-SG3: Investigate the relationship between personality and decision value. 
The aspects considered in this research are:

- Personality, one important human factor in decision-making. It can be measured for any personality instrument developed on psychology field of science;

- Decision-making process, related to group decision-making and part of the negotiation mentioned by Boehm and Jain [3] on Theory $\mathrm{W}$; and

- Decision value, related to the concept of value proposed by Boehm [2] and represents the perception of decision-value of each decision-maker.

Figure 1 illustrates the aspects studied in this research and how the specific goals are related to them.

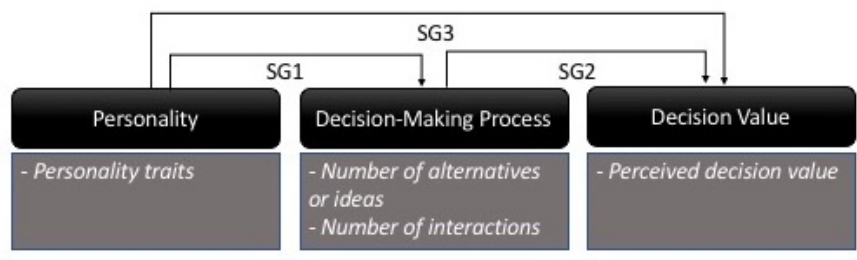

Figure 1: Aspects studied in this research and the related specific goals

The research methodology designed to reach the research goal is presented in the next section.

\section{RESEARCH METHODOLOGY}

Part of this PhD research was dedicated to conduct a systematic literature review that 15 articles that discuss the relationship between personality and decision making [5]. These articles employed most survey (about 53\%) and experiment (about 33\%) to study the abovementioned relationship. Because of these numbers, we concluded that these both methodologies (survey and experiment) are the most suitable for the type of research that we aim to conduct.

Furthermore, if we decide to conduct some observational studies in industry, for example, case study, we could have some problems: 1. Selection of cases that can fit the context of this research, i.e. companies that make group decision-making considering VBSE concepts; 2 . Company data privacy. Companies can just do not want to participate of this kind of research because we are investigating some sensitive company area, the way how companies make decisions.

Because of these abovementioned problems, and considering the research methodology that previous researches used, we chose experiment to conduct this research.

The experiment will revolve around a hypothetical software development company during the software development. Each instance of the experiment will have one group with four people, one for each of the following roles: customer, project manager, requirement engineer, and developer. The selection of the subjects will focus on people that work with software development and execute the same role of the experiment in their real life. These roles represent some key stakeholders who should participate in the decision-making process. It is important to highlight that the key stakeholders for each software project can variate however, this experiment will focus only in these four roles.
Before the experiment, the subjects will receive information about the company and about the project accordingly to the role they will play. They will also have their personality characterized before the experiment. During the experiment, the subjects will make some decisions related to the software development project during a meeting. The following data related to decision-making process will be collected: number of generated ideas/alternatives and number of interaction.

The decision value will come from the subjects after the experiment. We will ask individually how each subject evaluate the value of each decision made during the experiment. The concept of value will be explained before wards, according to Boehm definition [2]. Figure 1 presents the summary of the aspects studied in the experiment.These aspects were chosen by observing those studies included in the literature review conducted in this $\mathrm{PhD}$ research [5].

The hypothesis that will be explored in the experiment needs more studying, and this will be done during experiment planning phase. However, they will explore the interaction between the aspects we showed in Figure 1.

The data will be analyzed quantitatively aiming to verify what it is possible to conclude about the proposed hypothesis. A possible statistic technique to be employed is Partial Least Square or Multiple Linear Regression, according to the hypotheses that it will be defined.

\section{FINAL REMARKS}

The research presented in this document aims to explore the relationship between decision-making and personality on VBSE context. We chose experiment as a research method which will be based on a hypothetical scenario in which the subjects needs to make some decisions during a meeting.

From the theoretical perspective, the main contribution of this research is the understanding of the relationship between personality traits, the decision-making process, and decision value perception. The practitioners can use this research to improve their decisionmaking process, for example, by choosing the right people to make decisions based on personality traits.

At this moment of the $\mathrm{PhD}$, a manuscript related to a systematic literature review is under preparation [5].The next step of this $\mathrm{PhD}$ research is the planning of the experiment.

\section{REFERENCES}

[1] G Abatecola, G Mandarelli, and S Poggesi. 2013. The personality factor: how top management teams make decisions. A literature review. fournal of Management \& Governance 17, 4 (2013), 1073-1100.

[2] B Boehm. 2003. Value-based software engineering. ACM SIGSOFT Software Engineering Notes 28, 2 (2003), 3.

[3] B W Boehm and A Jain. 2006. An initial theory of value-based software engineering. In Value-Based Software Engineering. Springer, 15-37.

[4] S Cruz, F QB da Silva, and L F Capretz. 2015. Forty years of research on personality in software engineering: A mapping study. Computers in Human Behavior 46 (2015), 94-113.

[5] FF Mendes, E Mendes, and N Salleh. 2018. The relationship between personality and decision-making: a systematic literature review. (2018). Manuscript in preparation.

[6] N Salleh, Mendes FF, and E Mendes. 2017. A Systematic Mapping Study of Valuebased Software Engineering. (2017). Manuscript in preparation. 